\title{
Analysis of Multiterm Initial Value Problems with Caputo-Fabrizio Derivative
}

\author{
Mohammed Al-Refai ${ }^{1}{ }^{1}$ and Muhammed Syam $\mathbb{D}^{2}$ \\ ${ }^{1}$ Department of Mathematics, Yarmouk University, Irbid, Jordan \\ ${ }^{2}$ Department of Mathematical Sciences, UAE University, Al-Ain, UAE \\ Correspondence should be addressed to Mohammed Al-Refai; m_alrefai@yu.edu.jo
}

Received 6 August 2021; Revised 4 October 2021; Accepted 2 November 2021; Published 13 November 2021

Academic Editor: Antonio Di Crescenzo

Copyright (C) 2021 Mohammed Al-Refai and Muhammed Syam. This is an open access article distributed under the Creative Commons Attribution License, which permits unrestricted use, distribution, and reproduction in any medium, provided the original work is properly cited.

In this paper, we discuss the solvability of a class of multiterm initial value problems involving the Caputo-Fabrizio fractional derivative via the Laplace transform. We derive necessary and sufficient conditions to guarantee the existence of solutions to the problem. We also obtain the solutions in closed forms. We present two examples to illustrate the validity of the obtained results.

\section{Introduction}

Recently, there is great interest to develop new types of fractional derivatives of nonsingular kernel. Motivated by applications, Caputo and Fabrizio were the first to introduce such types of fractional derivatives with nonlocal and nonsingular kernel [1]. The Caputo-Fabrizio derivative is connected with a variety of applications (see [2-6]). Stability analysis of fractional differential equations without inputs was studied in [7], where exponential stability is obtained for the Caputo-Fabrizio derivative. Since their kernels are nonlocal, fractional derivatives preserve memories, and therefore, they have been used to model several (SIR) epidemic models (see [8-14]). Several analytical techniques have been implemented to study various fractional equations with fractional derivatives without singular kernels, such as the Laplace transform, reduction to initial value problems with integer derivatives, maximum principles, and fixed point theorems (see [15-21]), just to mention a few out of many in the literature. The following definitions are required to state our problem.

Definition 1. A function $f$ is said to be absolutely continuous on $[a, b]$, if there exists a function $f^{\prime} \in L^{1}(a, b)$ such that

$$
f(t)=f(a)+\int_{a}^{t} f^{\prime}(x) \mathrm{d} x, \quad \forall t \in[a, b] .
$$

In the following, we will use the notation $A C([a, b])$ to denote the space of absolutely continuous functions on $[a, b]$.

Definition 2. A function $f:[0,+\infty) \longrightarrow \mathbb{R}$ is said to be of exponential order, if there exists three constants $T, M, C \geq 0$ such that $|f(t)| \leq C e^{M t}, \forall t \geq T$.

Then, we define the following space.

Definition 3. The space $\mathscr{M}^{1}$ is defined by

$$
\mathscr{M}^{1}=\left\{f \in L_{\text {loc }}^{1}: f \text { is absolutely continuous on }[0, T] \text {, for any } T>0 \text {, and } f, f^{\prime} \text { are of exponential order }\right\} .
$$

In this paper, we consider the multiterm fractional initial value problem: 


$$
\begin{aligned}
\sum_{k=1}^{n} a_{k}\left({ }^{\mathrm{CFC}} D_{0}^{\alpha_{k}} y\right)(t) & =a_{n+1} y(t)+f(t), \quad t>0, n \geq 2, \\
y(0) & =y_{0},
\end{aligned}
$$

where $1>\alpha_{1}>\alpha_{2}>\cdots>\alpha_{n}>0, a_{k}>0, k=1, \ldots, n$, and $\left({ }^{\mathrm{CFC}} D_{0}^{\alpha}\right)$ is the Caputo-Fabrizio fractional derivative of Caputo sense. Here, we assume that $y, f \in \mathscr{M}^{1}$, so their Laplace transforms are well defined.

Definition 4 (see [1]). For $0<\alpha<1, t>0$ and $f \in \mathscr{M}^{1}$, the Caputo-Fabrizio fractional derivative of Caputo sense is defined by

$$
\left({ }^{\mathrm{CFC}} D_{0}^{\alpha} f\right)(t)=\frac{B(\alpha)}{1-\alpha} \int_{0}^{t} f^{\prime}(\tau) e^{-(\alpha /(1-\alpha))(t-\tau)} \mathrm{d} \tau,
$$

where $B(\alpha)>0$ is a normalization function satisfying $B(0)=B(1)=1$.

For the corresponding fractional integral and more properties of the derivative, we refer the readers to $[1,5,22-24]$. In $[15,17]$, the fractional initial value problems were transformed to equivalent initial value problems with integer derivatives. However, the technique is not valid for the multiterm initial value problems. The single term of the problem with $n=1$ was discussed in [23], and the solution of the problem was obtained in a closed form. In this paper, we apply the Laplace transform to analyze the solutions of the fractional initial value problems (3) and (4). This paper is organized as follows: in Section 2, we present some preliminary results about the Caputo-Fabrizio fractional derivative and derive necessary and sufficient conditions to guarantee the existence of solutions to problems (3) and (4). We also obtain the exact solutions in closed forms using the Laplace transform. In Section 3, we present two examples to illustrate the validity of our results. Finally, we close up with some concluding remarks in Section 4.

\section{Main Results}

We start with the definition and main results concerning the Caputo-Fabrizio derivative. Then, we present necessary and sufficient conditions for the solution of problems (3) and (4). Since $\quad\left({ }^{\mathrm{CFC}} D_{0}^{\alpha} f\right)(t)=(B(\alpha) /(1-\alpha)) f^{\prime}(t) * e^{-(\alpha /(1-\alpha)) t}$, equation (3) can be written as

$$
\sum_{k=1}^{n} c_{k} e^{-\mu_{k} t} * y^{\prime}(t)=c_{n+1} y(t)+f(t), \quad t>0, n \geq 2,
$$

where

$$
\begin{gathered}
c_{k}=\frac{B\left(\alpha_{k}\right)}{1-\alpha_{k}} a_{k}, \\
\mu_{k}=\frac{\alpha_{k}}{1-\alpha_{k}}, \quad k=1, \ldots, n, c_{n+1}=a_{n+1},
\end{gathered}
$$

and * denotes to the convolution of two functions.

Remark 1. Let $F(s)=\left(P_{n}(s) / Q_{m}(s)\right)$ where $P_{n}(s)$ and $Q_{m}(s)$ are polynomials with degrees $n$ and $m$, respectively, and are with real coefficients. If $n<m$, then the inverse Laplace transform of $F(s)$ exists and can be evaluated using partial fractions.

Lemma 1. For $f \in \mathscr{M}^{1}$, the following hold:

(1) ${ }^{\mathrm{CFC}} D_{0}^{\alpha}: A C([0, T]) \longrightarrow C([0, T])$

(2) $\left({ }^{\mathrm{CFC}} D_{0}^{\alpha} f\right)(0)=0$

Proof.

(1) For any function $f \in A C([0, T]),{ }^{C F C} D_{0}^{\alpha} f$ is the convolution product of a continuous function and $L^{1}$ function, that is continuous

(2) Since for any $f \in A C([0, T]),{ }^{C F C} D_{0}^{\alpha} f$ is continuous; then, ${ }^{\mathrm{CFC}} D_{0}^{\alpha} f(0)=\lim _{t \rightarrow 0^{+}}{ }^{\mathrm{CFC}} D_{0}^{\alpha} f(t)=0$

Definition 5. Let the space $\mathscr{M}^{2}$ be defined by

$$
\mathscr{M}^{2}=\left\{f \in C^{1}([0,+\infty)): f^{\prime}(0)=f^{\prime}\left(0^{+}\right) \in \mathbb{R}, f^{\prime} \in A C([0, T]), \text { for any } T>0 \text {, and } f, f^{\prime}, f^{\prime \prime} \text { are of exponential order }\right\}
$$

Lemma 2. The following holds for multiterm fractional initial value problems (3) and (4).

(1) If a solution $y:[0,+\infty) \longrightarrow \mathbb{R}$ exists such that $y \in A C([0, T])$ for any $T>0$, then $c_{n+1} y(0)+f(0)$ $=0$

(2) If $f \in \mathscr{M}^{1}, c_{n+1} y(0)+f(0)=0$, and $c_{n+1} \neq \sum_{k=1}^{n} c_{k}$, then a unique solution $y \in \mathscr{M}^{1}$ exists

(3) If a solution $y \in \mathscr{M}^{1}$ exists for $y_{0} \neq 0$ and $f \in \mathscr{M}^{2}$, then $c_{n+1} \neq \sum_{k=1}^{n} c_{k}$
Proof.

(1) Let $y \in C([0,+\infty))$ be a solution to multiterm fractional initial value problems (3) and (4) with $y \in A C([0, T])$ for any $T>0$. By Lemma 1 , ${ }^{\mathrm{CFC}} D_{0}^{\alpha_{k}} y(t)=0$ for any $k=1, \ldots, n$ Then, from equation (3), one gets

$$
c_{n+1} y(0)+f(0)=0 .
$$

(2) Let $y_{0} \neq 0, \quad f \in \mathscr{M}^{1}, c_{n+1} y(0)+f(0)=0$, and $c_{n+1} \neq \sum_{k=1}^{n} c_{k}$. Then, applying the Laplace transform 
to equation (6) and using the convolution result, we have

$$
\sum_{k=1}^{n} \frac{c_{k}}{s+\mu_{k}}\left[s \mathscr{L}(y)(t)-y_{0}\right]=c_{n+1} \mathscr{L}(y)(t)+F(s),
$$

where $F(s)=\mathscr{L}(f)(t)$. The above equation yields $\mathscr{L}(f)(t)\left(s \sum_{k=1}^{n} \frac{c_{k}}{s+\mu_{k}}-c_{n+1}\right)=y_{0} \sum_{k=1}^{n} \frac{c_{k}}{s+\mu_{k}}+F(s)$.

We have

$$
\begin{aligned}
\sum_{k=1}^{n} \frac{c_{k}}{s+\mu_{k}} & =\frac{c_{1}}{s+\mu_{1}}+\frac{c_{2}}{s+\mu_{2}}+\cdots+\frac{c_{n}}{s+\mu_{n}} \\
& =\sum_{k=1}^{n} c_{k} \frac{\prod_{j=1, j \neq k}^{n}\left(s+\mu_{j}\right)}{\prod_{k=1}^{n}\left(s+\mu_{k}\right)=\frac{Q_{n-1}(s)}{P_{n}(s)}}
\end{aligned}
$$

where $\quad Q_{n-1}(s)=\sum_{k=1}^{n} c_{k} \prod_{j=1, j \neq k}^{n}\left(s+\mu_{j}\right) \quad$ and $P_{n}(s)=\prod_{k=1}^{n}\left(s+\mu_{k}\right)$ are polynomials of degrees $n-$ 1 and $n$, respectively. Substituting in equation (11), we have

$\mathscr{L}(y)\left(s \frac{Q_{n-1}(s)}{P_{n}(s)}-c_{n+1}\right)=y_{0} \frac{Q_{n-1}(s)}{P_{n}(s)}+F(s)$,

or

$$
\begin{aligned}
\mathscr{L}(y)(s)= & y_{0} \frac{Q_{n-1}(s)}{s Q_{n-1}(s)-c_{n+1} P_{n}(s)} \\
& +\frac{P_{n}(s)}{s Q_{n-1}(s)-c_{n+1} P_{n}(s)} F(s) .
\end{aligned}
$$

Now, the leading coefficient of the polynomial $Q_{n-1}(s)$ is $\sum_{k=1}^{n} c_{k}$, and the leading coefficient of the polynomial $P_{n}(s)$ is 1 . If $c_{n+1} \neq \sum_{k=1}^{n} c_{k}$, then $s Q_{n-1}$ $(s)-c_{n+1} P_{n}(s)$ is a polynomial of degree $n$. Let

$$
\begin{aligned}
& H_{1}(s)=\frac{Q_{n-1}(s)}{s Q_{n-1}(s)-c_{n+1} P_{n}(s)}, \\
& H_{2}(s)=\frac{1}{s} \frac{P_{n}(s)}{s Q_{n-1}(s)-c_{n+1} P_{n}(s)},
\end{aligned}
$$

so that $\mathscr{L}^{-1}\left(H_{1}\right)(s)=h_{1}(t)$ and $\mathscr{L}^{-1}\left(H_{2}\right)(s)=h_{2}$ $(t)$ are well defined. Then,

$$
\begin{aligned}
\mathscr{L}(y)(t) & =y_{0} H_{1}(s)+H_{2}(s) s F(s) \\
& =y_{0} H_{1}(s)+H(s)\left(\mathscr{L}\left(f^{\prime}\right)(t)+f(0)\right) .
\end{aligned}
$$

Using the uniqueness result of the inverse Laplace transform, we have

$$
y(t)=y_{0} h_{1}(t)+h_{2}(t) * f^{\prime}(t)+f(0) h_{2}(t),
$$

which completes the proof.

(3) Let a solution $y \in \mathscr{M}^{1}$ exists for $y_{0} \neq 0$ and $f \in \mathscr{M}^{2}$. Starting from equation (14), let us define

$$
\begin{aligned}
\tilde{H}_{1}(s) & =y_{0} \frac{Q_{n-1}(s)}{s Q_{n-1}(s)-c_{n+1} P_{n}(s)}, \\
\tilde{g}(s) & =\frac{P_{n}(s)}{s Q_{n-1}(s)-c_{n+1} P_{n}(s)} F(s) \\
& =\frac{P_{n}(s)}{s^{2}\left(s Q_{n-1}(s)-c_{n+1} P_{n}(s)\right)} s^{2} F(s) .
\end{aligned}
$$

Define

$$
\tilde{H}_{2}(s)=\frac{P_{n}(s)}{s^{2}\left(s Q_{n-1}(s)-c_{n+1} P_{n}(s)\right)} .
$$

$\widetilde{H}_{2}$ admits an inverse Laplace transform since $s Q_{n-1}(s)-$ $c_{n+1} P_{n}(s)$ is of degree $n-1$. Moreover, since $f \in \mathscr{M}^{2}$, also $s^{2} F(s)$ admits an inverse Laplace transform, thus also $\tilde{g}(s)$ (by the convolution rule). One can rewrite equation (14) as

$$
\mathscr{L}(y)(s)-\widetilde{g}(s)=\widetilde{H}_{1}(s) .
$$

However, since $s Q_{n-1}(s)-c_{n+1} P_{n}(s)$ is of degree $n-1$ and $y \neq 0$, the right-hand side does not admit an inverse Laplace transform while the left-hand side does, which is a contradiction.

Lemma 3. Let $y \in A C([0, T]), T>0$, be a solution to the multiterm fractional initial value problem (3) with $y(0)=0$, then $f(0)=0$.

Proof. From Lemma 1 , since $y_{0}=y(0)=0$, then $f(0)=0$.

Remark 2. If $y(0)=f(0)=0, f \in \mathscr{M}^{1}$, and $c_{n+1} \neq \sum_{k=1}^{n} c_{k}$, then a solution $y \in \mathscr{M}^{1}$ exists by Lemma 2 . If $f \in \mathscr{M}^{2}$, a solution exists even if $c_{n+1}=\sum_{k=1}^{n} c_{k}$, and it can be evaluated using the Laplace transform in the following manner.

Lemma 4. Let $y(t)$ be a possible solution to multiterm fractional initial value problems (3) and (4), with $y(0)=f(0)=0, \quad c_{n+1}=\sum_{k=1}^{n} c_{k}, \quad$ and $f \in \mathscr{M}^{2}$. If $G(s)=s^{2} \mathscr{L}(f)(t)$ has an inverse Laplace transform, then $y(t)=h_{3}(t) * \mathscr{L}^{-1}\left(s^{2} F(s)\right)$, where $h_{3}(t)=\mathscr{L}^{-1}\left(H_{3}\right)(s)$ and $\mathrm{H}_{3}$ is defined in equation (22).

Proof. Substituting $y_{0}=0$, in equation (14), we have

$$
\mathscr{L}(y)(t)=\frac{P_{n}(s)}{s Q_{n-1}(s)-c_{n+1} P_{n}(s)} F(s) .
$$


Since $c_{n+1}=\sum_{k=1}^{n} c_{k}$, we have $s Q_{n-1}(s)-c_{n+1} P_{n}(s)$ is a polynomial of degree $n-1$. Let

$$
H_{3}(s)=\frac{1}{s^{2}} \frac{P_{n}(s)}{s Q_{n-1}(s)-c_{n+1} P_{n}(s)},
$$

so that $\mathscr{L}^{-1}\left(H_{3}\right)(s)=h_{3}(t)$ is well defined. Then, equation (14) yields

$$
\mathscr{L}(y)(t)=H_{3}(s) s^{2} F(s) .
$$
as

If $f \in \mathscr{M}^{2}$, then $\mathscr{L}(y)(t)$ in equation (23) can be written

$$
\begin{aligned}
\mathscr{L}(y)(t) & =H_{3}(s)\left(\mathscr{L}\left(f^{\prime \prime}\right)(t)+s f(0)+f^{\prime}(0)\right) \\
& =H_{3}(s)\left(\mathscr{L}\left(f^{\prime \prime}\right)(t)+f^{\prime}(0)\right) .
\end{aligned}
$$

Applying the inverse Laplace transform, we have

$$
y(t)=h_{3} * f^{\prime \prime}(t)+f^{\prime}(0) h_{3}(t)
$$

We summarize the obtained results in the following main theorem.

Theorem 1. Consider multiterm fractional initial value problems (3) and (4).

(1) For $y_{0}=0$, if a solution $y \in A C([0, T]), T>0$, exists, then $f(0)=0$

(2) If $f \in \mathscr{M}^{1}, c_{n+1} y(0)+f(0)=0$, and $c_{n+1} \neq \sum_{k=1}^{n} c_{k}$, then a solution $y \in \mathscr{M}^{1}$ exists

(3) If $f \in \mathscr{M}^{2}$ and $y_{0} \neq 0$, then a solution $y \in \mathscr{M}^{1}$ exists if and only if $c_{n+1}=\sum_{k=1}^{n} c_{k}$

(4) If $y_{0}=0, f \in \mathscr{M}^{2}$, and $f(0)=0$, then a solution $y \in \mathscr{M}^{1}$ exists

\section{Illustrative Examples}

We discuss two main examples. The first one is a two-term problem where it holds that $c_{n+1}=\sum_{k=1}^{n} c_{k}$. We show the existence of a solution for a specific case by imposing extra conditions. The second example is a three-term problem where it holds that $c_{n+1} \neq \sum_{k=1}^{n} c_{k}$. So, the existence of a unique solution is guaranteed. We present the solution in a closed form and discuss several special cases.

Example 1. Consider the two-term fractional initial value problem:

$$
\begin{aligned}
\left(e^{-\mu_{1} t}+e^{-\mu_{2} t}\right) * y^{\prime}(t) & =2 y(t)+f(t) \\
y(0) & =y_{0}
\end{aligned}
$$

Since $c_{1}+c_{2}=c_{3}$, then the problem has no solution for $y(0) \neq 0$. For $y(0)=f(0)=0$ and $s^{2} \mathscr{L}(f)(t)$ has Laplace inverse, the problem admits a solution. To verify, let us find the solution given by equation (25). We have

$$
\begin{aligned}
Q_{1}(s) & =2 s+\mu_{1}+\mu_{2}, \\
P_{2}(s) & =\left(s+\mu_{1}\right)\left(s+\mu_{2}\right), \\
H_{3}(s) & =-\frac{1}{s^{2}} \frac{\left(s+\mu_{1}\right)\left(s+\mu_{2}\right)}{\left(\mu_{1}+\mu_{2}\right) s+2 \mu_{1} \mu_{2}} \\
& =\frac{R_{1}}{s}+\frac{R_{2}}{s^{2}}+\frac{R_{3}}{\left(\mu_{1}+\mu_{2}\right) s+2 \mu_{1} \mu_{2}},
\end{aligned}
$$

where

$$
\begin{aligned}
& R_{1}=-\frac{1}{4} \frac{\mu_{1}+\mu_{2}}{\mu_{1} \mu_{2}}, \\
& R_{2}=-\frac{1}{2}, \\
& R_{3}=\frac{1}{4} \frac{\left(\mu_{1}-\mu_{2}\right)^{2}}{\mu_{1} \mu_{2}} .
\end{aligned}
$$

Thus,

$$
h_{3}(t)=\mathscr{L}^{-1}\left(H_{3}\right)(s)=R_{1}+R_{2} t+\frac{R_{3}}{\mu_{1}+\mu_{2}} e^{-2\left(\mu_{1} \mu_{2} / \mu_{1}+\mu_{2}\right) t},
$$

and the solution $y(t)=h_{3}(t)^{*} f^{\prime \prime}(t)+f^{\prime}(0) h_{3}(t)$ is obtained, provided that $f \in \mathscr{M}^{2}$.

As a special case, let us consider $f(t)=t^{2}$. Then, $f(0)=0, \mathscr{L}(f)(t)=\left(2 / s^{3}\right)$, and $s^{2} \mathscr{L}(f)(t)=(2 / s)$ have Laplace inverse. Thus,

$$
\begin{aligned}
y(t) & =h_{3}(t) * f^{\prime \prime}(t)+f^{\prime}(0) h_{3}(t)=h_{3}(t) * 2 \\
& =2 \int_{0}^{t}\left(R_{1}+R_{2} \tau+\frac{R_{3}}{\mu_{1}+\mu_{2}} e^{-2\left(\mu_{1} \mu_{2} / \mu_{1}+\mu_{2}\right) \tau}\right) \mathrm{d} \tau \\
& =2 R_{1} t+R_{2} t^{2}-\frac{R_{3}}{\mu_{1} \mu_{2}}\left(e^{-2\left(\mu_{1} \mu_{2} / \mu_{1}+\mu_{2}\right)}-1\right) .
\end{aligned}
$$

For $\mu_{1}=(2 / 3)$ and $\mu_{2}=(1 / 3)$, we have $R_{1}=-(9 / 8), R_{2}=-(1 / 2), R_{3}=(1 / 8)$, and

$$
y(t)=\frac{9}{4} t-\frac{1}{2} t^{2}-\frac{9}{16} e^{-(4 / 9) t}+\frac{9}{16} .
$$

Example 2. As a second example, we consider the threeterm initial value problem:

$$
\begin{aligned}
\left(e^{-\mu_{1} t}+e^{-\mu_{2} t}+e^{-\mu_{3} t}\right) * y^{\prime}(t) & =y(t)+f(t), \\
y(0) & =y_{0} .
\end{aligned}
$$

Since $c_{1}=c_{2}=c_{3}=c_{4}=1$ and $c_{1}+c_{2}+c_{3} \neq c_{4}$, the problem has a unique solution given by equation (17) provided that $y(0)+f(0)=0$. We have 


$$
\begin{aligned}
Q_{2}(s)=\sum_{k=1}^{3} c_{k} \prod_{j=1, j \neq k}^{3}\left(s+\mu_{j}\right)= & \left(s+\mu_{2}\right)\left(s+\mu_{3}\right) \\
& +\left(s+\mu_{1}\right)\left(s+\mu_{3}\right) \\
& +\left(s+\mu_{1}\right)\left(s+\mu_{2}\right), P_{3}(s) \\
= & \prod_{k=1}^{3}\left(s+\mu_{k}\right) \\
= & \left(s+\mu_{1}\right)\left(s+\mu_{2}\right)\left(s+\mu_{3}\right) .
\end{aligned}
$$

Let

$$
T_{3}(s)=s Q_{2}(s)-c_{4} P_{3}(s)=2 s^{3}+\left(\mu_{1}+\mu_{2}+\mu_{3}\right) s^{2}-\mu_{1} \mu_{2} \mu_{3} \text {, }
$$

then

$$
\begin{aligned}
& H_{1}(s)=\frac{Q_{2}(s)}{T_{3}(s)}, \\
& H_{2}(s)=\frac{1}{s} \frac{P_{3}(s)}{T_{3}(s)},
\end{aligned}
$$

with

$$
\begin{aligned}
& \mathscr{L}^{-1}\left(H_{1}\right)(s)=h_{1}(t), \\
& \mathscr{L}^{-1}\left(H_{2}\right)(s)=h_{2}(t),
\end{aligned}
$$

and the solution is given by

$$
y(t)=y_{0} h_{1}(t)+h_{2}(t) * f^{\prime}(t)+f(0) h_{2}(t) .
$$

For $f(t)=t$, we have $y(0)=-f(0)=0$, and thus

$$
y(t)=h_{2}(t) * 1=\int_{0}^{t} h_{2}(\tau) \mathrm{d} \tau .
$$

For $f(t)=1$, we have $y(0)=-f(0)=-1$, and thus

$$
y(t)=h_{2}(t)-h_{1}(t)
$$

As a specific case, if we consider $\mu_{1}=(7 / 5), \mu_{2}=(1 / 2)$, and $\mu_{3}=(1 / 3)$, we have

$$
\begin{aligned}
& Q_{2}(s)=3 s^{2}+\frac{67}{15} s+\frac{4}{3}, \\
& P_{3}(s)=s^{3}+\frac{67}{30} s^{2}+\frac{4}{3} s+\frac{7}{30}, \\
& T_{3}(s)=2 s^{3}+\frac{67}{30} s^{2}-\frac{7}{30}, \\
& H_{1}(s)=\frac{Q_{2}(s)}{P_{3}(s)}=\frac{90 s^{2}+134 s+40}{60 s^{3}+67 s^{2}-7}=\frac{A_{0}}{s+1}+\frac{A_{1}}{s+\Delta_{1}}+\frac{A_{2}}{s+\Delta_{2}}, \\
& H_{2}(s)=\frac{P_{3}(s)}{s T_{3}(s)}=\frac{30 s^{3}+67 s^{2}+40 s+7}{s(s+1)\left(s+\Delta_{1}\right)\left(s+\Delta_{2}\right)}=-\frac{1}{s}+H_{1}(s),
\end{aligned}
$$

where $\Delta_{1}=(7+\sqrt{1729}) / 120, \Delta_{2}=(7-\sqrt{1729}) / 120, A_{0}=$ $-(2 / 23), A_{1}=(73 \sqrt{1729}-3113) / 92 \sqrt{1729}, A_{2}=$
$(73 \sqrt{1729}+3113) / 92 \sqrt{1729}, \quad h_{1}(t)=A_{0} e-t+A_{1} e^{-\Delta_{1} t}$ $+A_{2} e^{-\Delta_{2} t}$, and $h_{2}(t)=h_{1}(t)-1$.

For $f(t)=t$, we have $y(t)=\int_{0}^{t} h_{2}(\tau) \mathrm{d} \tau=\left(A_{0}+\left(A_{1} / \Delta_{1}\right)\right.$ $\left.+\left(A_{2} / \Delta_{2}\right)\right)-t-A_{0} e^{-t}-\left(A_{1} / \Delta_{1}\right) e^{-\Delta_{1} t}-\left(A_{2} / \Delta_{2}\right) e^{-\Delta_{2} t}$.

\section{Concluding Remarks}

We obtained the solutions of a class of multiterm fractional initial value problems in closed forms using the Laplace transform. We have also discussed several necessary and sufficient conditions to guarantee the existence of solutions to the problem. Whether the results are extendable to wider classes of multiterm initial value problems or systems of fractional equations is left for future work.

\section{Data Availability}

The data used to support the findings of this study are included within the article.

\section{Conflicts of Interest}

The authors declare that there are no conflicts of interest.

\section{Authors' Contributions}

The first author has initiated the idea and obtained the analytical results. The second author did the numerical examples, shared the discussion, and approved the final draft of the manuscript.

\section{References}

[1] M. Caputo and M. Fabrizio, "A new definition of fractional derivative without singular kernel," Progress in Fractional Differentiation and Applications, vol. 1, pp. 73-85, 2015.

[2] B. S. T. Alkahtani and A. Atangana, "Controlling the wave movement on the surface of shallow water with the CaputoFabrizio derivative with fractional order," Chaos, Solitons \& Fractals, vol. 89, pp. 539-546, 2016.

[3] A. Atangana and J. J. Nieto, "Numerical solution for the model of RLC circuit via the fractional derivative without singular kernel," Advances in Mechanical Engineering, vol. 7, pp. 1-7, 2015.

[4] A. Atangana, "On the new fractional derivative and application to nonlinear Fisher's reaction-diffusion equation," Applied Mathematics and Computation, vol. 273, pp. 948-956, 2016.

[5] M. Caputo and M. Fabrizio, "Applications of new time and spatial fractional derivatives with exponential kernels," Progress in Fractional Differentiation and Applications, vol. 2, no. 1, pp. 1-11, 2016.

[6] M. Caputo and M. Fabrizio, "Deformations in elasto-plastic media with memory: the inverse problem," Progress in Fractional Differentiation and Applications, vol. 4, no. 1, pp. 5-14, 2018.

[7] N. Sene, "Stability analysis of the fractional differential equations with the Caputo-Fabrizio fractional derivative," Journal of Fractional Calculus and Applications, vol. 1, pp. 160-172, 2020. 
[8] O. A. Arqub and A. El-Ajou, "Solution of the fractional epidemic model by homotopy analysis method," Journal of King Saud University Science, vol. 25, no. 1, pp. 73-81, 2013.

[9] B. Acay, E. Bas, and T. Abdeljawad, "Fractional economic models based on market equilibrium in the frame of different type kernels," Chaos, Solitons \& Fractals, vol. 130, Article ID 109438, 2020.

[10] Z. Chen, K. Liu, X. Liu, and Y. Lou, "Modelling epidemics with fractional-dose vaccination in response to limited vaccine supply," Journal of Theoretical Biology, vol. 486, Article ID 110085, 2020.

[11] M. Khan and A. Atangana, "Modeling the dynamics of novel coronavirus (2019-nCov) with fractional derivative," Alexandria Engineering Journal, vol. 4, no. 59, pp. 2379-2389, 2020.

[12] H. Kheiri and M. Jafari, "Fractional optimal control of an HIV/AIDS epidemic model with random testing and contact tracing," Journal of Applied Mathematics and Computing, vol. 60 , no. $1-2$, pp. $387-411,2019$.

[13] D. Kumar and J. Singh, "New aspects of fractional epidemiological model for computer viruses with mittag-leffler law," in Mathematical Modelling in Health, Social and Applied Sciences. Forum for Interdisciplinary Mathematics, H. Dutta, Ed., Springer, Singapore, 2020.

[14] N. Sene, "SIR epidemic model with Mittag-Leffler fractional derivative," Chaos, Solitons \& Fractals, vol. 137, Article ID 109833, 2020.

[15] M. Al-Refai, "Reduction of order formula and fundamental set of solutions for linear fractional differential equations," Applied Mathematics Letters, vol. 82, pp. 8-13, 2018.

[16] M. Al-Refai and K. Pal, "A maximum principle for a fractional boundary value problem with convection term and applications," Mathematical Modeling and Analysis, vol. 24, no. 1, pp. 62-71, 2019.

[17] M. Al-Refai and K. Pal, "New aspects of Caputo-Fabrizio fractional derivative," Progress in Fractional Differentiation and Applications, vol. 5, no. 2, pp. 157-166, 2019.

[18] E. Karimov and S. Pirnafasov, "Higher order multi-term timefractional partial differential equations involving CaputoFabrizio derivative," The Electronic Journal of Differential Equations, vol. 243, pp. 1-11, 2017.

[19] H. Marasi, A. Joujehi, and H. Aydi, "An Extension of the Picard theorem to fractional differential equations with a Caputo-Fabrizio derivative," Journal of Function Spaces, vol. 2021, Article ID 6624861, 6 pages, 2021.

[20] N. Sene, "Stokes' first problem for heated flat plate with Atangana-Baleanu fractional derivative," Chaos, Solitons \& Fractals, vol. 117, pp. 68-75, 2018.

[21] A. Shaikh, A. Tassaddiq, K. S. Nisar, and D. Baleanu, "Analysis of differential equations involving Caputo-Fabrizio fractional operator and its applications to reaction-diffusion equations," Advances in Difference Equations, vol. 2019, no. 1, Article ID 178, 2019.

[22] T. Abdeljawad and D. Baleanu, "On fractional derivatives with exponential kernel and their discrete versions," Reports on Mathematical Physics, vol. 80, no. 1, pp. 11-27, 2017.

[23] J. Losada and J. Nieto, "Properties of a new fractional diffusion equations with fractional derivative of non-singular kernel," Progress in Fractional Differentiation and Applications, vol. 1, no. 2, pp. 87-92, 2015.

[24] M. Al-Refai, "Proper inverse operators of fractional derivatives with nonsingular kernels," Rendiconti del Circolo Matematico di Palermo Series 2, vol. 2021, 2021. 